\title{
Human platelet lysate as an alternative to fetal bovine serum for culture and endothelial differentiation of human amniotic fluid mesenchymal stem cells
}

\author{
WALEEPHAN TANCHAROEN $^{1}$, SIRINDA AUNGSUCHAWAN ${ }^{1}$, \\ PERAPHAN POTHACHAROEN ${ }^{2}$, KANOKKAN BUMROONGKIT ${ }^{1}$, CHANIPORN PUANINTA $^{1}$, \\ NATHAPORN PANGJAIDEE ${ }^{1}$, SUTEERA NARAKORNSAK ${ }^{1}$, RUNCHANA MARKMEE ${ }^{1}$, \\ TANONGSAK LAOWANITWATTANA $^{1}$ and CHAWAPON THAOJAMNONG ${ }^{1}$ \\ ${ }^{1}$ Department of Anatomy, Faculty of Medicine, Chiang Mai University; \\ ${ }^{2}$ Thailand Excellence Center for Tissue Engineering and Stem Cells, Department of Biochemistry, \\ Faculty of Medicine of Chiang Mai University, Chiang Mai 50200, Thailand
}

Received October 12, 2018; Accepted April 2, 2019

DOI: $10.3892 / \mathrm{mmr} .2019 .10182$

\begin{abstract}
Human amniotic fluid (hAF) mesenchymal stem cells (MSCs) are commonly cultured in medium containing FBS. However, there are concerns about using animal serum in therapeutic applications due to the potential for immunogenic reactions and the risk of transmission of pathogens. For safety reasons, human platelet lysate (hPL) has been suggested as a replacement for FBS because it appears to be a natural source of growth factors. In this present study, it was investigated whether FBS could be substituted with hPL in hAF-MSCs culture without affecting their properties. Pooled hPL was generated by the freeze-thaw method. The concentration of hPL was selected after evaluation by MTT assay. The hAF-MSCs were cultured in FBS- or hPL-supplemented conditions and shared a fibroblast-like morphology. Cell proliferation assays showed that the growth characteristic of hAF-MSCs cultured in 10\% hPL-supplemented media was similar to those cultured in $10 \%$ FBS-supplemented media. The expression of MSC markers did not differ between the cells cultured in the different conditions. The endothelial differentiation potential was also investigated. Reverse transcription-quantitative (RT-q)PCR revealed that induced cells supplemented with hPL showed an increase level of endothelial specific gene expression compared to the FBS-supplemented cells. Immunofluorescence analysis showed specific protein localization in both induced cell
\end{abstract}

Correspondence to: Dr Sirinda Aungsuchawan, Department of Anatomy, Faculty of Medicine, Chiang Mai University, 110 Maharaj Nakorn Chiang Mai Hospital, Intawarorose Road, A. Muang, Chiang Mai 50200, Thailand

E-mail: sirinda001@hotmail.com

Key words: amniotic fluid, endothelial cells, fetal bovine serum, human platelet lysate, mesenchymal stem cells groups. Additionally, induced cells supplemented with hPL had the potential to form networks on Matrigel. This present study indicated that hPL could be used to culture and enhance the endothelial differentiation potential of hAF-MSCs.

\section{Introduction}

Mesenchymal stem cells (MSCs) are viewed as a promising tool for cell-based therapies. Amniotic fluid is a source of mesenchymal stem cells that is of increasing interest. Human amniotic fluid (hAF) MSCs can be isolated from amniotic fluid from the second trimester of pregnancy by amniocentesis, a minimally invasive procedure. hAF-MSCs account for $0.9-1.5 \%$ of the cellular population in the amniotic fluid $(1,2)$. As hAF-MSCs can give rise to osteogenic, chondrogenic, adipogenic, myogenic, endothelial, hepatic or neurogenic lineages (3) they have been shown to be effective in the treatment of many diseases (4). Due to the endothelial differentiation potential of MSCs, they have received a significant amount of attention as an autologous source for cell-based therapy in restoring endothelial functions and promoting vascularization $(5,6)$.

In the cultivation process, the isolation and expansion protocols use FBS as a supplement as it has been used in several clinical trials (7). While FBS contains various growth factors, hormones and nutrients (8), it also possesses a high endotoxin content and can be a potential source of microbial contaminants, including fungi, bacteria, viruses and prions. Thus, clinical trials and future therapeutic applications of human MSCs should aim to avoid the use serums derived from animals (9). Studies have raised concerns about the possibility of animal pathogen transmission during the culture and transplantation processes $(7,10)$. Additionally, it has been reported that patients who have received cell transplantations with MSCs expanded in FBS exhibit antibodies against bovine antigens (11). Therefore, a variety of human supplements have been selected as alternatives to the use of FBS to provide nutrients and growth factors (12). 
Human platelet lysate (hPL) has been proposed as an alternative to animal serum for the expansion of MSCs in vitro (13). hPL contains various growth factors, including platelet-derived growth factor (PDGF), transforming growth factor (TGF) and epidermal growth factor (EGF) (11). These growth factors have been shown to enhance the proliferation rate of MSCs and maintain their multilineage differentiation potential under cultivation in the absence of FBS (9). Bioactive molecules and growth factors contained in hPL have been shown to support the expansion of MSCs derived from bone marrow (BM) (12), umbilical cord blood (14) and adipose tissue (10). Additionally, hPL has been reported to induce the endothelial differentiation of BM-MSCs (15).

Based on relevant data $(9,10,12,14,15)$, the present study investigated the use of FBS or hPL as a supplement for cell culture and compared the characteristics of hAF-MSCs under these conditions. This present study focused on the endothelial differentiation potential of AF-MSCs when they were induced with vascular endothelial growth factor (VEGF) supplemented with either FBS or hPL.

\section{Materials and methods}

Preparation of human platelet lysate. Human donor platelets $(n=15)$ were obtained from the blood bank of Maharaj Nakorn Chiang Mai Hospital using the platelet apheresis method after positive red blood cell antibody screening. Subsequently, hPL was prepared in accordance with a previously described method (8). Briefly, 15 pooled groups of platelets were frozen at $-80^{\circ} \mathrm{C}$ and then thawed at $37^{\circ} \mathrm{C}$; this was repeated three times. To remove membrane fragments, the lysate was centrifuged at $2,200 \mathrm{x} \mathrm{g}$ at room temperature for $20 \mathrm{~min}$ and the supernatant was filtered through a $0.2 \mu \mathrm{m}$ filter (Corning Inc.). Aliquots of the platelet lysate were stored at $-20^{\circ} \mathrm{C}$. To avoid hPL gel formation, $2 \mathrm{U} / \mathrm{ml}$ heparin (LEO Pharma A/S) was added as an anticoagulant.

MTT cell viability assay. MTT (cat. no. 298-93-1; Sigma-Aldrich; Merck KGaA) was used to evaluate the optimal concentrations of hPL. hAF-MSCs were plated in a 96 well-plate at $5 \times 10^{3}$ cells in triplicate and incubated at $37^{\circ} \mathrm{C}$ with $5 \% \mathrm{CO}_{2}$ and $95 \%$ humidity for $24 \mathrm{~h}$. The cells were cultured with DMEM-high glucose (Gibco; Thermo Fisher Scientific, Inc.) supplemented with hPL (2.5, 5, 10, 20 or $40 \%$ ) for 24,48 or $72 \mathrm{~h}$. As a control, cells were cultured with DMEM alone, containing neither FBS nor hPL. At the indicated time points, medium was removed and replaced with MTT solution $(0.5 \mathrm{mg} / \mathrm{ml}$ in DMEM). After a further $4 \mathrm{~h}$ of incubation under the same conditions as for culture, MTT solution was removed and $100 \mu \mathrm{l}$ DMSO was added to dissolve the formazan crystals. The absorbance was determined at $540 \mathrm{~nm}$ with a spectrophotometer.

Cell preparation and culture. Human amniotic fluid cell samples with a normal karyotype were obtained during weeks 16-22 of gestation from the Human Genetics Laboratory of the Anatomy Department, Faculty of Medicine, Chiang Mai University. This study was approved by the Research Ethics Committee of the Faculty of Medicine, Chiang Mai University on March 13th 2018 (no. ANA-2561-05343).
The cells collected were cultured with BIOAMF-3 $3^{\mathrm{TM}}$ Complete Medium (Biological Industries) in a $25 \mathrm{~cm}^{2}$ culture flask (Corning Inc.) at $37^{\circ} \mathrm{C}, 5 \% \mathrm{CO}_{2}$ and $95 \%$ humidity for 9 days at the Human Genetics Laboratory for routine prenatal diagnosis. After obtaining the cells, the medium was removed and the cells were washed with sterile PBS. They were then detached from the flask with $0.25 \%$ trypsin-EDTA (Gibco; Thermo Fisher Scientific, Inc.) and cultured in the basic growth medium: DMEM-high glucose with gentamycin, penicillin and streptomycin, and supplemented with FBS (cat. no. 16000036; Gibco; Thermo Fisher Scientific, Inc.) or hPL. The basic growth medium was supplemented with either $10 \%$ FBS, 10\% hPL or $20 \% \mathrm{hPL}$. The cell density of each group was adjusted to $10^{5}$ cells $/ \mathrm{cm}^{2}$ in the culture flask. Cells were then incubated at $37^{\circ} \mathrm{C}, 5 \% \mathrm{CO}_{2}$ and $95 \%$ humidity. The medium was changed every 3 days. Upon reaching $80 \%$ confluence, the passage 1 adherent cells were detached using $0.25 \%$ trypsin-EDTA. The determination of cell proliferation and characterization was performed after two passages.

Cell proliferation. hAF-MSCs were plated on a 24 well-plate (Corning Inc.) at a density of $10^{4}$ cells $/ \mathrm{cm}^{2}$. They were then cultured in basic media with either $10 \% \mathrm{FBS}, 10 \% \mathrm{hPL}$ or $20 \%$ hPL (10\% FBS was used as the control in this experiment) at $37^{\circ} \mathrm{C}, 5 \% \mathrm{CO}_{2}$ and $95 \%$ humidity. Cells were counted on day 0 and day 1 , and then every second day until day 21 after seeding using a hemocytometer.

Flow cytometry. To characterize hAF-MSCs, the cell surface markers of cells cultured in basic media containing 10\% FBS or $10 \%$ hPL were evaluated. The cells were trypsinized with $0.25 \%$ trypsin-EDTA at $37^{\circ} \mathrm{C}$ for $1 \mathrm{~min}$ and centrifuged at $2,035 \mathrm{x} \mathrm{g}$ for $6 \mathrm{~min}$ at room temperature to obtain the cell pellets. Then, non-specific binding was blocked using $10 \%$ human $\mathrm{AB}$ serum [serum from type $\mathrm{AB}$ donors; processed by our laboratory and inactivated at $53^{\circ} \mathrm{C}$ for $90 \mathrm{~min}$ as previously described (16)] at $4^{\circ} \mathrm{C}$ for $30 \mathrm{~min}$. Subsequently, they were incubated with the following monoclonal antibodies: Mouse anti-human CD34-FITC (cat. no. 343504; BioLegend, Inc.), CD44-FITC (cat. no. 21810443; ImmunoTools GmbH), CD45-phycoerythrin (PE; cat. no. 304008; BioLegend, Inc.), CD73-PE (cat. no. 21270734; ImmunoTools GmbH), HLA-ABC-FITC (cat. no. 21159033; ImmunoTools GmbH) and HLA-DR-PE (cat. no. 21388994; ImmunoTools GmbH). Cell surface marker expression was detected using a FACScan (BD Biosciences) and analyzed using CellQuest ${ }^{\mathrm{TM}}$ Pro 9.0 software (BD Biosciences).

Differentiation of hAF-MSCs into endothelial cells. To investigate the endothelial differentiation potential of hAF-MSCs, the cells were plated in basic medium supplemented with $10 \%$ FBS or $10 \%$ hPL at a density of $10^{4}$ cells $/ \mathrm{cm}^{2}$ in a $24-w e l l$ plate and induced with VEGF (cat. no. V7259; Sigma-Aldrich; Merck KGaA) for 14 days. They were then sub-cultured into two groups and cultured in basic media supplemented with either $10 \% \mathrm{FBS}$ and $50 \mathrm{ng} / \mathrm{ml} \mathrm{VEGF}$ or with $10 \% \mathrm{hPL}$ and $50 \mathrm{ng} / \mathrm{ml}$ of $\operatorname{VEGF}(\mathrm{n}=5)$.

Reverse transcription quantitative $(R T-q) P C R$. Total RNA of cells (FBS-supplemented non-induced cells, 
Table I. Primer sequences of reverse transcription-quantitative PCR.

\begin{tabular}{ll}
\hline Primer & \multicolumn{1}{c}{ Sequence } \\
\hline vWF & \\
Forward 5'-3' & CAAGGAAGAAAATAACACAGGTGAA \\
Reverse 5'-3' & TCATTGACCTTGCAGAAGTGAGTAT \\
VEGFR2 & \\
Forward 5'-3' & GACTTCCTGACCTTGGAGCATCT \\
Reverse 5'-3' & GATTTTAACCACGTTCTTCTCCGA \\
eNOS & \\
Forward 5'-3' & TCCCCCAGAACTCTTCCTT \\
Reverse 5'-3' & CTCATTCTCCAGGTGCTTCA \\
GAPDH & \\
Forward 5'-3' & ATGGGGAAGGTGAAGGTCG \\
Reverse 5'-3' & TAAAAGCAGCCCTGGTGACC \\
\hline
\end{tabular}

vWF, von Willebrand factor; VEGFR2, vascular endothelial growth factor receptor 2; eNOS endothelial nitric oxide synthase.

hPL-supplemented non-induced cells, FBS-supplemented induced cells and hPL-supplemented induced cells; $n=5$ ) was extracted using an Illutra RNAspin Mini RNA Isolation kit (GE Healthcare Life Sciences). First strand complementary DNA (cDNA) was then synthesized from total RNA using a Tetro cDNA synthesis kit (cat. no. BIO-65043; Bioline), according to the manufacturer's instructions. Briefly, the samples were incubated at $45^{\circ} \mathrm{C}$ for $30 \mathrm{~min}$, followed by $85^{\circ} \mathrm{C}$ for $5 \mathrm{~min}$ to terminate the reaction. Gene transcripts were measured using a SensiFAST ${ }^{\mathrm{TM}}$ SYBR $^{\circledR}$ No-ROX kit (cat. no. BIO-98005; Bioline) with a 7500 Fast Real-Time PCR System (Applied Biosystems; Thermo Fisher Scientific, Inc.). PCR primers targeting von Willebrand Factor (vWF), VEGF receptor 2 (VEGFR2), and endothelial nitric oxide synthase (eNOS) were used (Table I) (17). The protocol consisted of 36 cycles of $30 \mathrm{sec}$ of denaturation at $95^{\circ} \mathrm{C}, 30 \mathrm{sec}$ of annealing at $60^{\circ} \mathrm{C}$ and $60 \mathrm{sec}$ of extension at $72^{\circ} \mathrm{C}$. GAPDH was used as an internal control. The expression level of endothelial specific genes was plotted using the $2^{-\Delta \Delta C q}$ method (18).

Immunofluorescence. Cells from all conditions (non-induced and induced cells with FBS or hPL supplement; $n=5$ ) were analyzed for the expression of endothelial specific markers (vWF VEGFR2, and eNOS). In brief, the cells were washed twice with PBS and fixed with $4 \%$ paraformaldehyde at room temperature for $15 \mathrm{~min}$. They were then permeabilized with $0.2 \%$ Triton X-100 (Amresco, LLC) in PBS at room temperature for $10 \mathrm{~min}$. Then, blocking for non-specific binding was conducted with $10 \%$ human AB serum in $1 \%$ BSA-PBS for $30 \mathrm{~min}$ at room temperature, followed by incubation with mouse monoclonal antibody against human vWF (cat. no. MA5-14029; Pierce; Thermo Fisher Scientific, Inc.), rabbit monoclonal antibody against human VEGFR2 (cat. no. MA5-16417; Pierce; Thermo Fisher Scientific, Inc.) or mouse monoclonal antibody against eNOS (cat. no. MA5-15559; Pierce; Thermo Fisher Scientific, Inc.) at $37^{\circ} \mathrm{C}$ for $2 \mathrm{~h}$ at a dilution of 1:200.

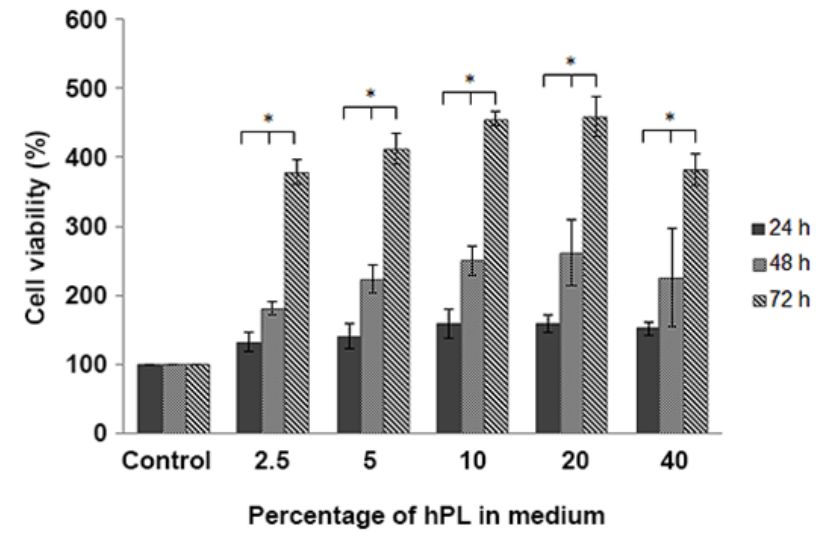

Figure 1. Determination of hAF-MSC cell viability following culture in hPL using the MTT assay. Viability of hAF-MSCs cultured in media supplemented with various concentrations of hPL for 24,48 and $72 \mathrm{~h}$. The cells cultured in media with no supplementation were used as the control. The percentage of viable cells is presented as the mean \pm SEM. The Kruskal-Wallis test with post hoc Dunn's test analysis were used to analyze the results of this experiment $\left({ }^{*} \mathrm{P}<0.05\right)$. hAF-MSCs, human amniotic fluid mesenchymal stem cells; hPL, human platelet lysate.

After washing the cells twice with PBS, FITC-conjugated goat anti-mouse IgG antibody (cat. no. 62-6511; Thermo Fisher Scientific, Inc.) or PE-conjugated goat anti-rabbit IgG antibody (cat. no. P-2771MP; Thermo Fisher Scientific, Inc.) were added and incubated at $37^{\circ} \mathrm{C}$ for $1 \mathrm{~h}$ at a dilution of 1:500. For nuclear staining, the cells were incubated with antifade DAPI $(2 \mu 1)$ for $10 \mathrm{~min}$ at room temperature (Invitrogen; Thermo Fisher Scientific, Inc.). Samples were examined using an Olympus AX70 fluorescent microscope and images were captured with DP manager and DP controller (magnification, $\mathrm{x} 20$; Olympus Corporation). Statistical analysis of fluorescent signals was conducted using Image J 1.50i software (National Institutes of Health).

Network formation. Network formation potential was assessed by incubating the cells from all conditions (non-induced and induced cells with FBS or hPL supplement; $n=5$ ) in Matrigel growth factor-reduced basement membrane matrix (Corning Inc.), according to the manufacturer's instructions. Briefly, Matrigel was thawed at $4{ }^{\circ} \mathrm{C}$ overnight and added to a 96 well-plate at a concentration of $50 \mu 1 / \mathrm{cm}^{2}$ with pre-cooled pipette tips. Next, the plate was incubated at $37^{\circ} \mathrm{C}$ for $30 \mathrm{~min}$. After cell preparation, cells were counted and the concentration was adjusted to $2 \times 10^{5}$ cells $/ 100 \mu \mathrm{l}$. Cells were then added at the selected density to the gel-coated wells and were incubated at $37^{\circ} \mathrm{C}, 5 \% \mathrm{CO}_{2}$ and $95 \%$ humidity for $24 \mathrm{~h}$. A human umbilical vein endothelial cell (HUVEC) line (Gibco; Thermo Fisher Scientific, Inc.) was used as a positive control. Network formation was observed under a light microscope (magnification, $\mathrm{x} 20$ ). ImageJ angiogenesis analyzer (http://image.bio.methods. free.fr/ImageJ/?Angiogenesis-Analyzer-for-ImageJ) was used to analyze the total mesh area and the mesh number.

Statistical analysis. Data were presented as the mean \pm standard error of the mean ( $\mathrm{n}=5$ for all experiments). Statistical comparisons were performed three times using a Kruskal-Wallis test with a post hoc Dunn's test or a Mann-Whitney U test. 


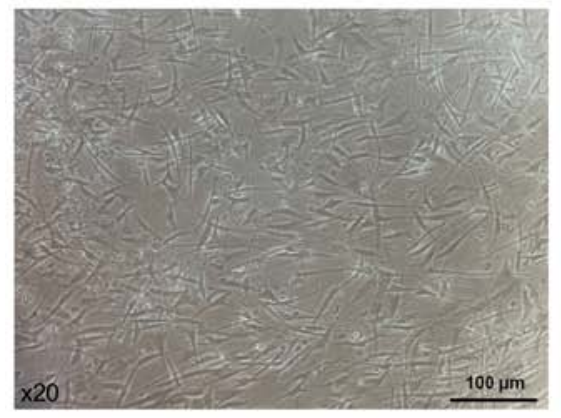

$10 \%$ FBS

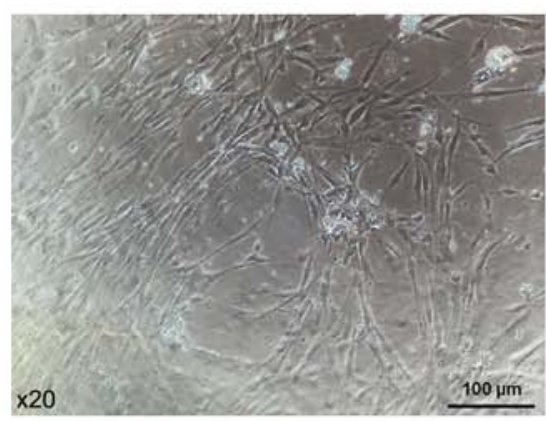

$10 \% \mathrm{hPL}$

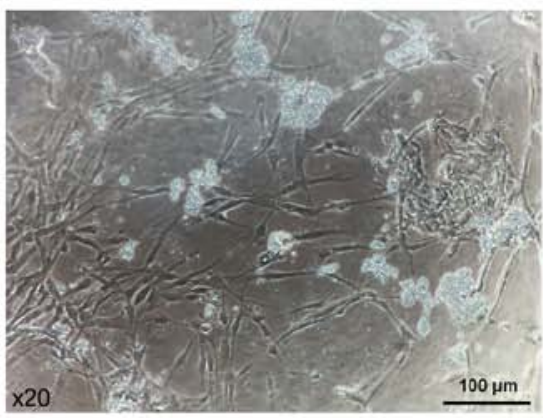

$20 \% \mathrm{hPL}$

Figure 2. Morphology of the hAF-MSCs cultured in media supplemented with FBS or hPL. hAF-MSCs cultured in $10 \%$ FBS-supplemented medium, $10 \%$ hPL-supplemented medium or $20 \%$ hPL-supplemented medium (magnification, x20; scale bar, $100 \mu \mathrm{m}$ ). hAF-MSCs, human amniotic fluid mesenchymal stem cells; hPL, human platelet lysate.

Statistical analysis was performed using SPSS version 22.0 software (IBM Corp). $\mathrm{P}<0.05$ was considered to indicate a statistically significant difference.

\section{Results}

Analysis of cell viability using the MTT assay. hAF-MSCs were cultured in basic media supplemented with hPL at 2.5, $5,10,20$ or $40 \%$ for 24,48 and $72 \mathrm{~h}$. The results indicated an increase in cell viability compared to the control at all time points. Cell viability at $72 \mathrm{~h}$ was significantly increased compared with at 24 and $48 \mathrm{~h}$ under all experimental conditions, with the exception of control treatment $(\mathrm{P}<0.05$; Fig. 1).

Morphology of hAF-MSCs. Microscopic examination revealed that at passage 2 the hAF-MSCs cultured in basic media supplemented with $10 \% \mathrm{FBS}, 10 \% \mathrm{hPL}$ or $20 \% \mathrm{hPL}$ densely adhered to the flask in a monolayer and displayed a homogeneous fibroblast-like morphology (Fig. 2).

Proliferation of hAF-MSCs. The growth curve demonstrated that cell number in each condition (Fig. 3) increased continuously in the log phase with no significant difference between conditions. The 10\% FBS- and 10\% hPL-supplemented cells entered into the stationary phase with no significant differences in cell number. However, cells supplemented with $20 \%$ hPL showed a decrease in cell number between days 11 and 15 . A lower cell number was maintained between days 13 and 21 .

Analysis of the expression of cell surface markers by flow cytometry. Flow cytometry was used to characterize the expression of cell surface markers in the hAF-MSCs. The results showed that the expression of cell surface markers did not differ between the cells cultured in 10\% FBS-supplemented media and $10 \% \mathrm{hPL}$-supplemented media. The hAF-MSCs from these two groups were positive for CD44, CD73 and HLA-ABC, cell surface markers that are expressed by hAF-MSCs and negative for CD34, CD45 and HLA-DR (Fig. 4).

Morphology of induced hAF-MSCs. hAF-MSCs were induced and divided into two groups (basic media supplemented with $10 \% \mathrm{FBS}$ and $50 \mathrm{ng} / \mathrm{ml}$ VEGF or basic media supplemented

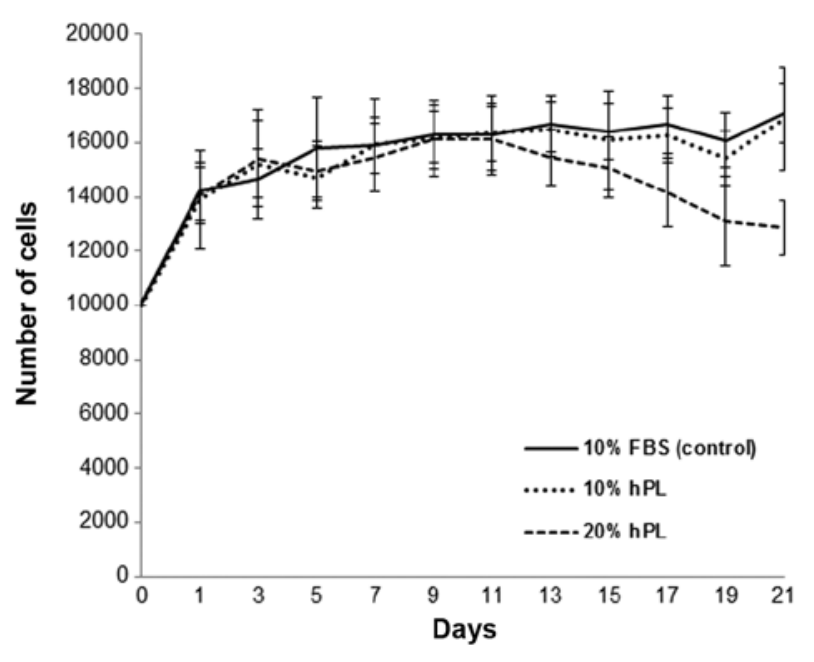

Figure 3. Proliferation of hAF-MSCs cultured in media supplemented with FBS or hPL. Number of hAF-MSCs cultured in media supplemented with either $10 \%$ FBS, $10 \%$ hPL or $20 \%$ hPL. Data are presented as the mean \pm SEM. The Kruskal-Wallis test and a post hoc analysis were used to analyze the results of this experiment. hAF-MSCs, human amniotic fluid mesenchymal stem cells; hPL, human platelet lysate.

with $10 \% \mathrm{hPL}$ and $50 \mathrm{ng} / \mathrm{ml}$ VEGF). After 14 days of VEGF exposure in media supplemented with either $10 \%$ FBS or $10 \%$ hPL, both sets of cells exhibited a spindle shape. However, oval and round spaces appeared within the surrounding adhered cells in the hAF-MSCs cultured with the hPL-supplemented media (Fig. 5).

Detection of endothelium-related gene expression. After 14 days of induction with VEGF, the expression of endothelium-related genes, including vWF, VEGFR2 and eNOS, was determined using RT-qPCR (Fig. 6). The results revealed that the expression levels of vWF (Fig. 6A), VEGFR2 (Fig. 6B) and eNOS (Fig. 6C) were increased in FBS-supplemented induced cells when compared to non-induced control cells cultured in FBS supplemented media (2.66-fold, 2.10-fold and 1.73 -fold, respectively). Similarly, the levels of these genes were increased in the hPL-supplemented induced cells when compared to non-induced control cells cultured in hPL supplemented media (3.93-fold, 2.94-fold and 2.30-fold, respectively). Furthermore, the expression levels of $v W F$, 

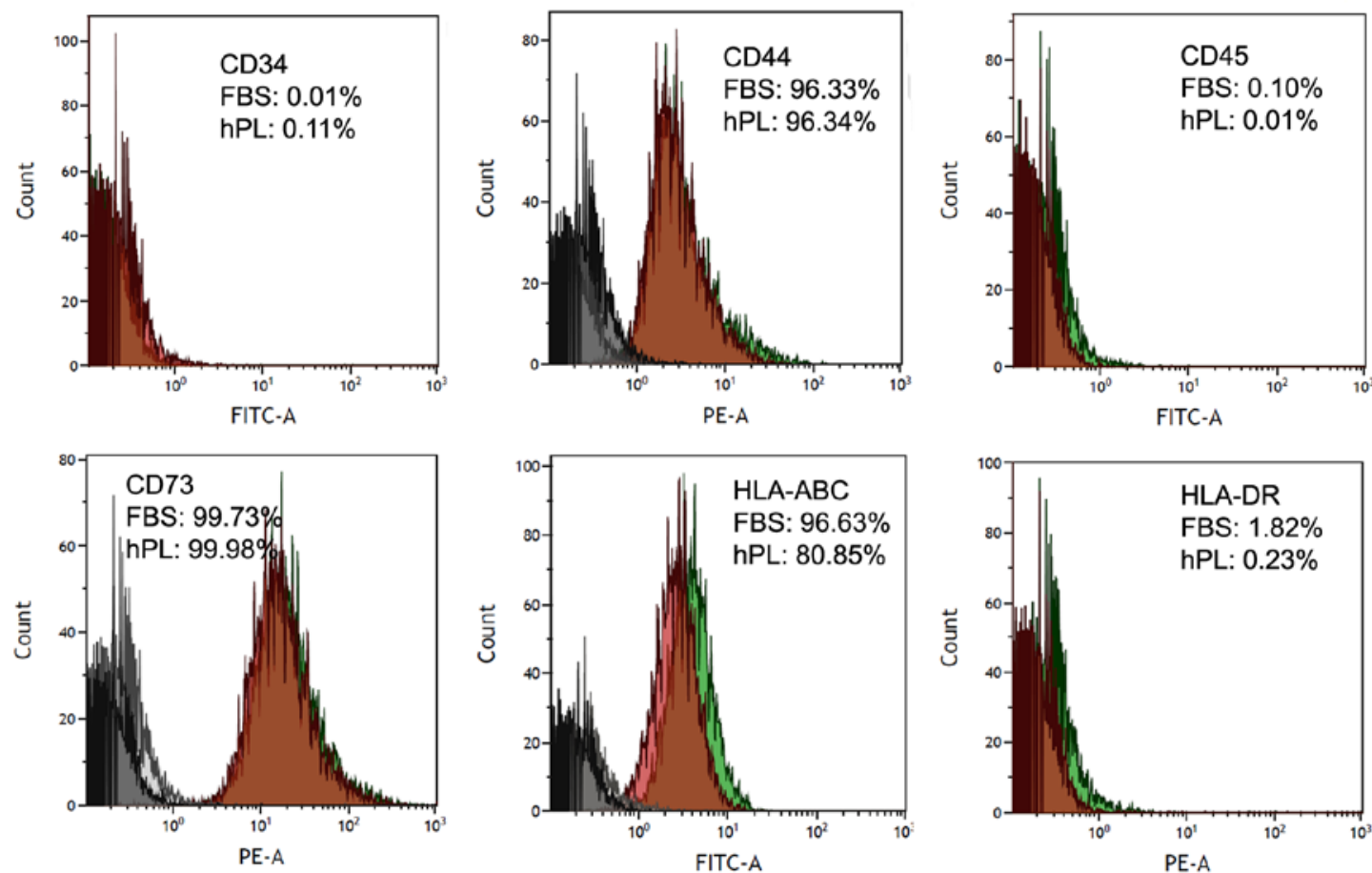

Figure 4. Analysis of cell surface marker expression in hAF-MSCs cultured in media supplemented with FBS or hPL. Flow cytometry histograms of $10 \%$ FBS-supplemented (green) or 10\% hPL-supplemented (red) hAF-MSCs with isotype control (black: FBS-supplemented cells; gray: hPL-supplemented cells). The expression of the cell surface markers CD34, CD44, CD45, CD73, HLA-ABC and HLA-DR were analyzed. hAF-MSCs, human amniotic fluid mesenchymal stem cells; hPL, human platelet lysate.

$V E G F R 2$ and $e N O S$ were increased to a greater extent in the induced cells that were cultured with $10 \%$ hPL compared to the cells that had been cultured with $10 \%$ FBS.

Expression of endothelial specific markers. The expression of vWF, VEGFR2 and eNOS was analyzed under both induced and non-induced conditions. The results of immunofluorescence analysis revealed that the induced cells cultured with either FBS or hPL expressed vWF, VEGFR2 and eNOS. Conversely, no signal was detected for these proteins in non-induced conditions when cultured in media supplemented with either FBS or hPL (Fig. 7A). Analysis using ImageJ $1.50 \mathrm{i}$ software was used to calculate the corrected total cell fluorescence (CTCF). The data showed that there were significant differences in CTCF levels for vWF (Fig. 7B), VEGFR2 (Fig. 7C) and eNOS (Fig. 7D) between the non-induced and induced cells when cultured with either FBS or hPL. CTCF analysis revealed no marked differences between induced cells cultured in FBS-supplemented or hPL-supplemented media (Fig. 7B-D).

Ability to form networks. The formation of networks was observed using a light microscope after incubating the cells in Matrigel-coated plates for $24 \mathrm{~h}$. The non-induced cells from both the FBS and hPL conditions did not present a network-like structure, while the FBS-supplemented induced cells exhibited some connection to the cell processes. Interestingly, the hPL-supplemented induced cells displayed a network formation that was similar to HUVECs (Fig. 8A).
The quantitative data were analyzed by angiogenesis analyzer, Image J $1.50 \mathrm{i}$ software. The data are presented in terms of the total mesh area (Fig. 8B) and mesh number (Fig. 8C) in FBS-supplemented induced cells, hPL-supplemented induced cells and HUVECs. The Kruskal-Wallis test and post hoc analysis demonstrated that the quantification of the two parameters between three groups was found to be significant.

\section{Discussion}

MSCs have been used in cell-based therapies to treat or restore damaged tissue (4). Amniotic fluid is a source of MSCs in which the cells can be isolated and expanded easily (19). Although FBS is widely used to culture MSCs, this culturing method raises concerns about the potential for xenogeneic protein transmission from the animal-derived sera (13). This present study investigated pooled hPL as a replacement for FBS in the culture media of hAF-MSCs, with a focus on the potential of hAF-MSCs to undergo endothelial differentiation. The hPL was obtained from platelet concentrate via a standardized platelet apheresis technique, providing a high concentration of platelets and a low level of leukocyte contamination (20). The optimal concentration of hPL was evaluated using the MTT assay. The results revealed significant differences after hAF-MSCs were cultured in hPL-supplemented media for $72 \mathrm{~h}$; however, there was no significant difference in terms of cell viability at each concentration. Based on this data, 10 and $20 \%$ hPL were selected in order to investigate the mesenchymal characteristics of hAF-MSCs. 


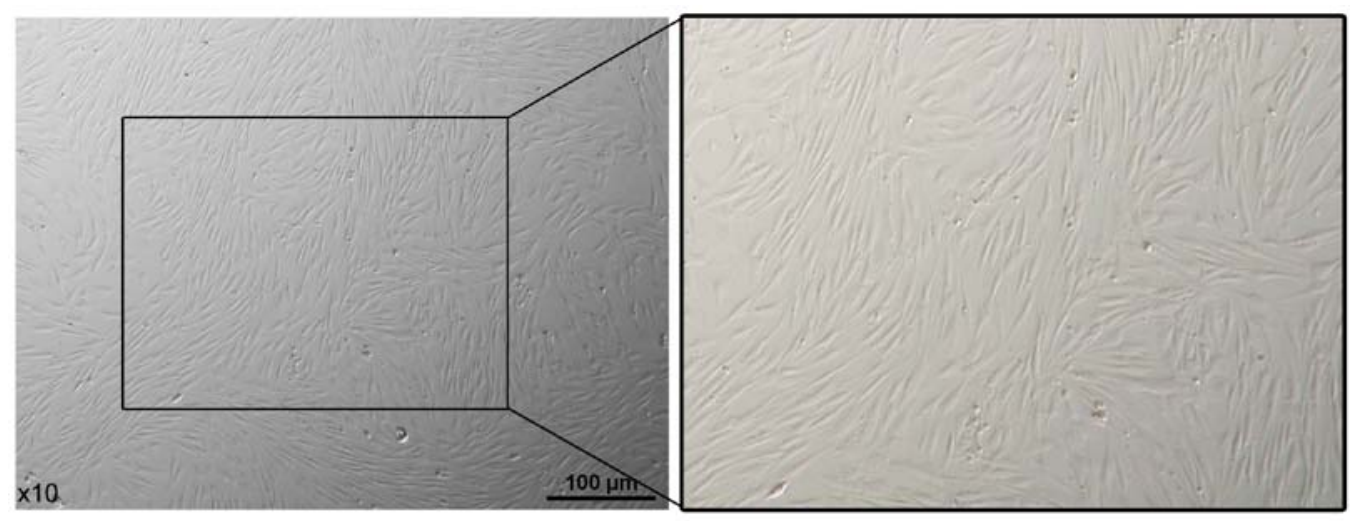

Induced (10\% FBS)

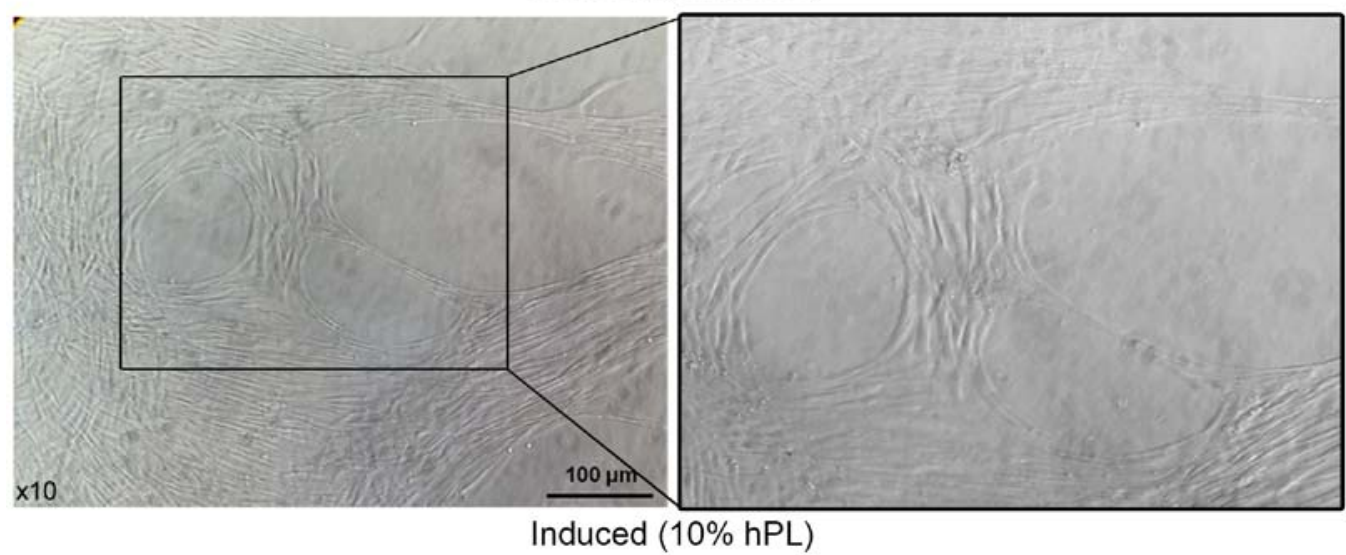

Figure 5. Morphology of hAF-MSCs cultured in media supplemented with FBS or hPL. The morphology of 10\% FBS-supplemented or 10\% hPL-supplemented hAF-MSCs induced with vascular endothelial growth factor for 14 days (left: Magnification, x10, scale bar, 100 $\mu$ m; right: Magnification, x40). hAF-MSCs, human amniotic fluid mesenchymal stem cells; hPL, human platelet lysate.
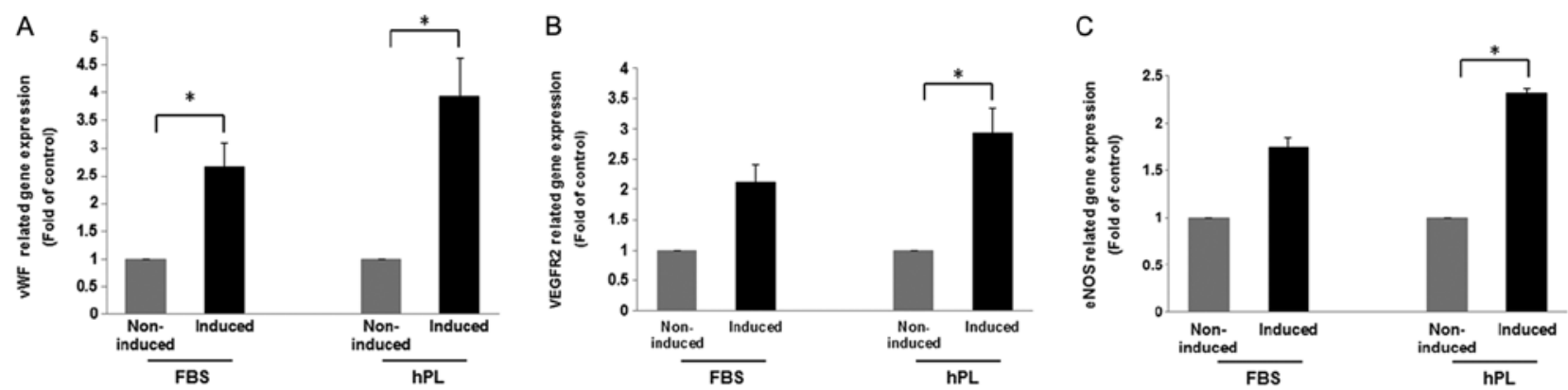

Figure 6. Detection of endothelial-related gene expression in non-induced and induced human amniotic fluid mesenchymal stem cells by real time quantitative PCR. FBS-supplemented non-induced cells, hPL-supplemented non-induced cells, FBS-supplemented induced cells and hPL-supplemented induced cells were analyzed to determine endothelial-related gene expression levels. The relative expressions of (A) vWF, (B) VEGFR2 and (C) eNOS was determined. Gene expression levels were normalized to GAPDH and were relative to the control. The Mann-Whitney U Test was performed to analyze the results of this experiment ${ }^{*} \mathrm{P}<0.05$. hPL, human platelet lysate; vWF, von Willebrand factor; VEGFR2, vascular endothelial growth factor receptor 2; eNOS endothelial nitric oxide synthase.

The culture of hAF-MSCs with media supplemented with $10 \%$ FBS, $10 \%$ hPL or $20 \%$ hPL revealed a fibroblast-like morphology, consistent with previous studies $(10,11,21)$. This present study found that the culturing of hAF-MSCs for 21 days in 10\% FBS- or 10\% hPL-supplemented media revealed no significant differences in terms of proliferation capacity. However, proliferation capacity decreased in cells cultured with $20 \%$ hPL; this result was not consistent with a previous study (22). This may suggest variations in the components of hPL between batches. Studies have revealed that there are many growth factors contained in hPL that promote MSC proliferation and differentiation, including basic fibroblast growth factor (bFGF), EGF, insulin-like growth factor 1 (IGF-1), PDGF, VEGF and TGF- $\beta(7,9,23)$. As has been reported previous studies, hPL is often used at a concentration of $10 \%(9,24,25)$. Consequently, this concentration was selected to evaluate MSC characteristics. The results of the flow cytometry experiments indicated that hAF-MSCs cultured with $10 \%$ FBS or $10 \%$ hPL had a similar level of expression of cell surface markers. The data presented here indicate that 
A
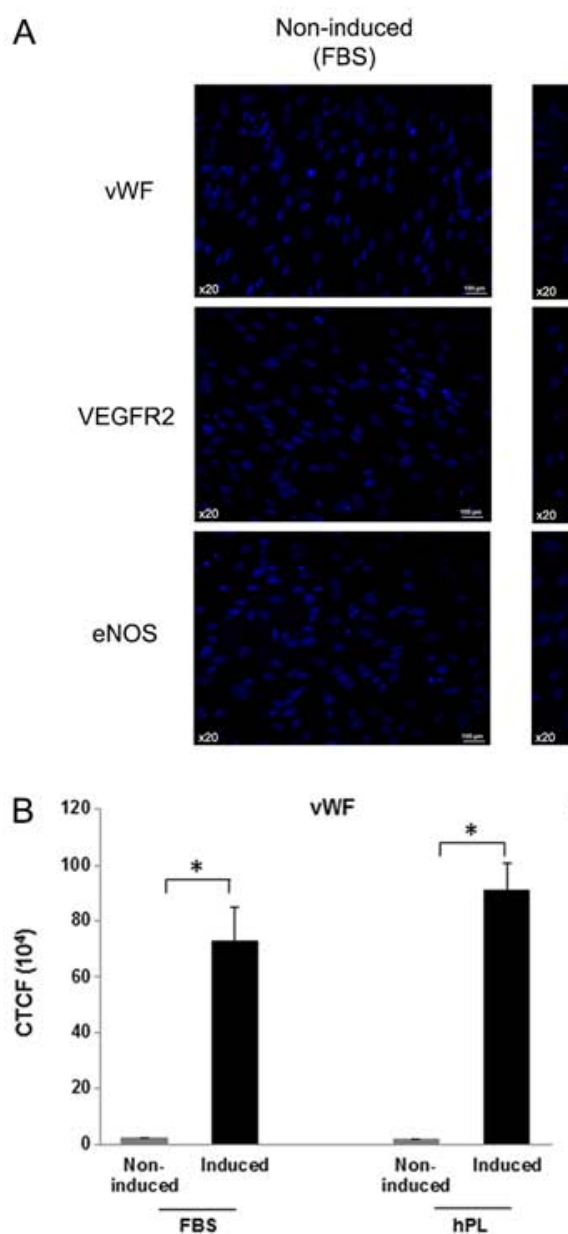

Non-induced (hPL)
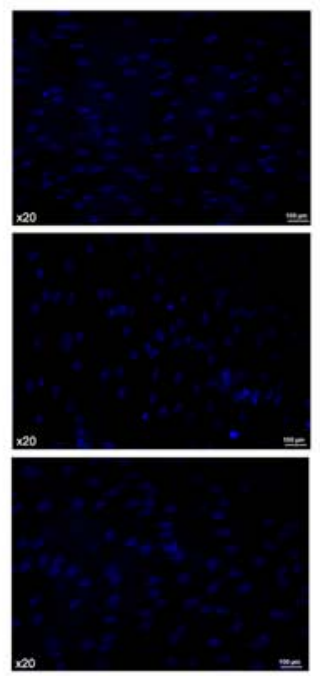
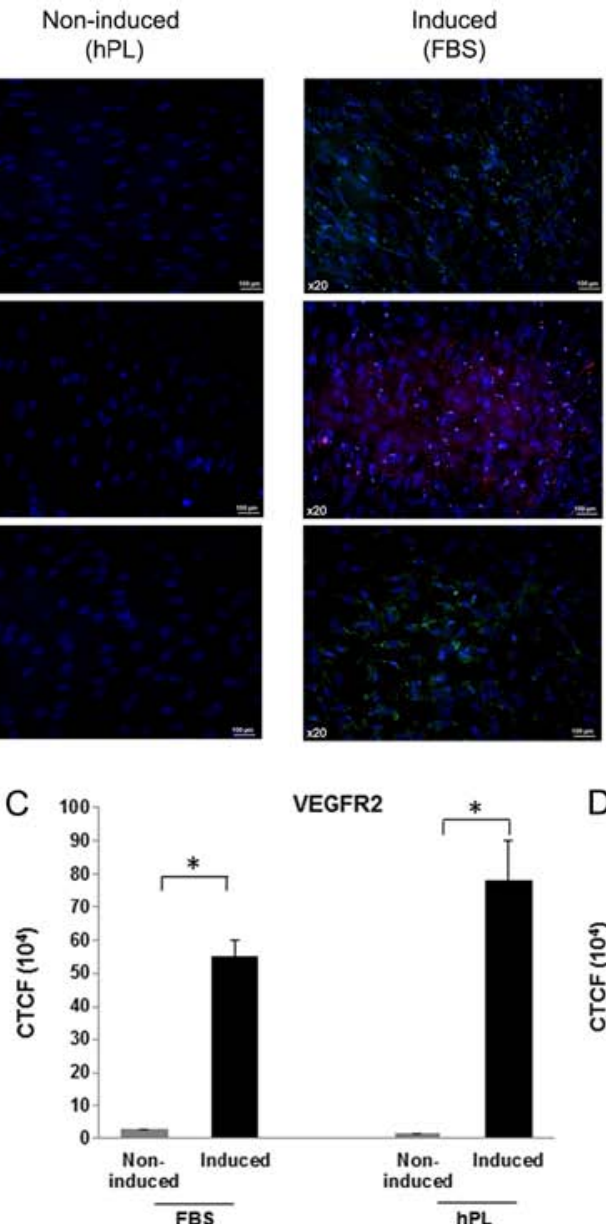
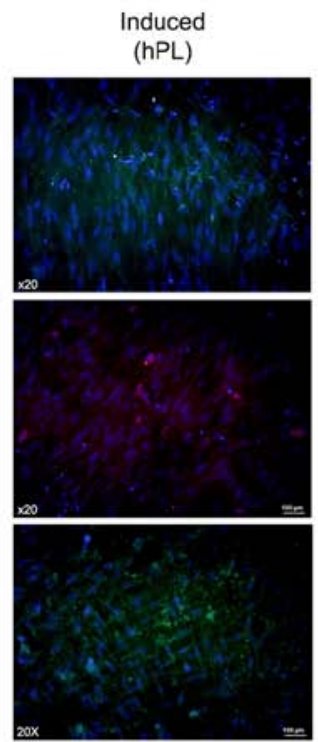

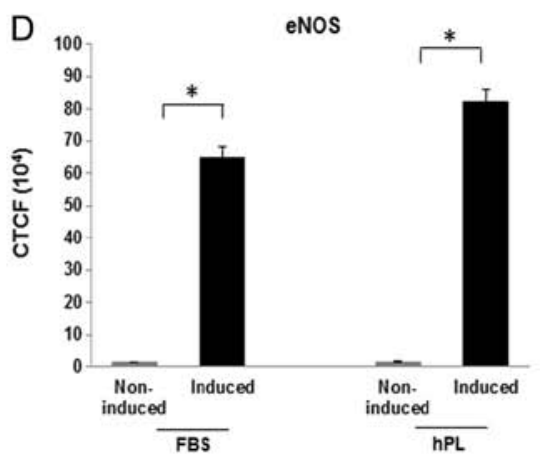

Figure 7. Detection of the expression of endothelial-specific markers using immunofluorescence. FBS-supplemented non-induced cells, hPL-supplemented non-induced cells, FBS-supplemented induced cells and hPL-supplemented induced cells were stained with antibodies against vWF, VEGFR2 or eNOS. Cell nuclei were stained with DAPI (magnification, x20; scale bar, $100 \mu \mathrm{m}$ ). (A) Representative images of cells stained for vWF, VEGFR2 and eNOS. Quantification of fluorescent signals using corrected total cell fluorescence of (B) vWF, (C) VEGFR2 and (D) eNOS. The Mann-Whitney U test was used to analyze the results of this experiment "P<0.05. hPL, human platelet lysate; vWF, von Willebrand factor; VEGFR2, vascular endothelial growth factor receptor 2; eNOS endothelial nitric oxide synthase.

supplementation of media with hPL can maintain the growth characteristics and the phenotypic profile of hAF-MSCs (26). Therefore, there is the possibility that hPL can be used instead of FBS in pre-clinical applications.

According to the properties of MSCs, they are able to differentiate according to their mesodermal lineage. Previous studies have shown the potential of hAF-MSCs to be differentiated into osteocytes and chondrocytes $(27,28)$. In this present study, hAF-MSCs that were induced using VEGF when cultured with hPL-supplemented media were capable of endothelial differentiation. Following 14 days of stimulation by VEGF, the hAF-MSCs displayed endothelial-like characteristics. The induced cells cultured in media supplemented with FBS were densely adhered with a fibroblast-like morphology. By contrast, the cells cultured in media supplemented with hPL displayed different arrangements, consistent with another study (29). Gene expression analysis using RT-qPCR also demonstrated that vWF, VEGFR2 and eNOS were expressed in induced cells cultured in media supplemented with either FBS or hPL at higher levels than in the non-induced cells. Furthermore, the data indicated that the cells cultured with hPL and induced with VEGF showed a higher level of expression than induced cells cultured with FBS. These results were investigated further using immunofluorescence analysis. This analysis indicated that there were specific proteins expressed in induced cells cultured with FBS or hPL. These results were consistent with a previous study that reported that hPL can induce expression of endothelial markers (15). Platelet lysate has been identified as a potent induction factor because it contains various growth factors that have an effect on endothelial cell maturation and growth (15). It has been reported that VEGF and angiopoietin-1 in hPL stimulate the AKT pathway to promote cell survival and vascular development in cardiovascular function (30). Studies have also indicated that supplementation of media with IGF-1, EGF and bFGF can enhance the endothelial differentiation potential of induced cells (31-33).

This present study also indicates that induced hAF-MSCs have the potential to form networks on Matrigel. hPL-supplemented induced cells formed network-likes structure that were comparable with those formed by HUVECs. This suggested that growth factors released by platelets could promote angiogenesis in vitro. Studies have demonstrated that hPL induces vasculogenic and angiogenic responses in endothelial 
A

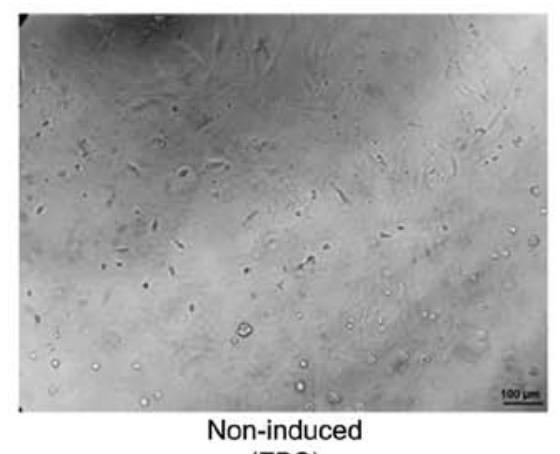

(FBS)

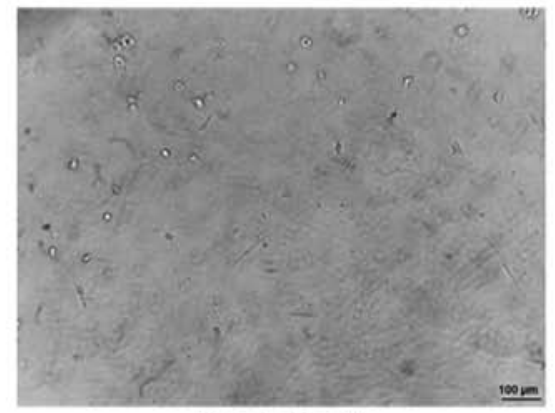

Non-induced

(hPL)

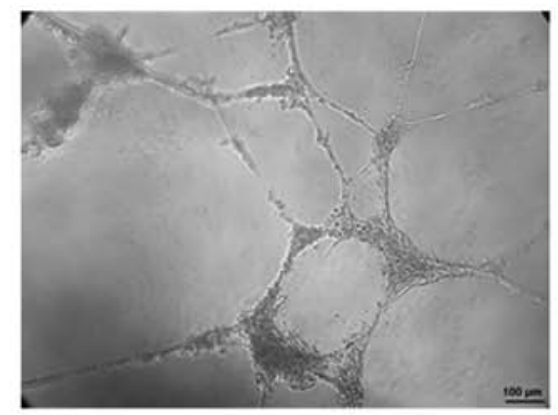

HUVECS

B

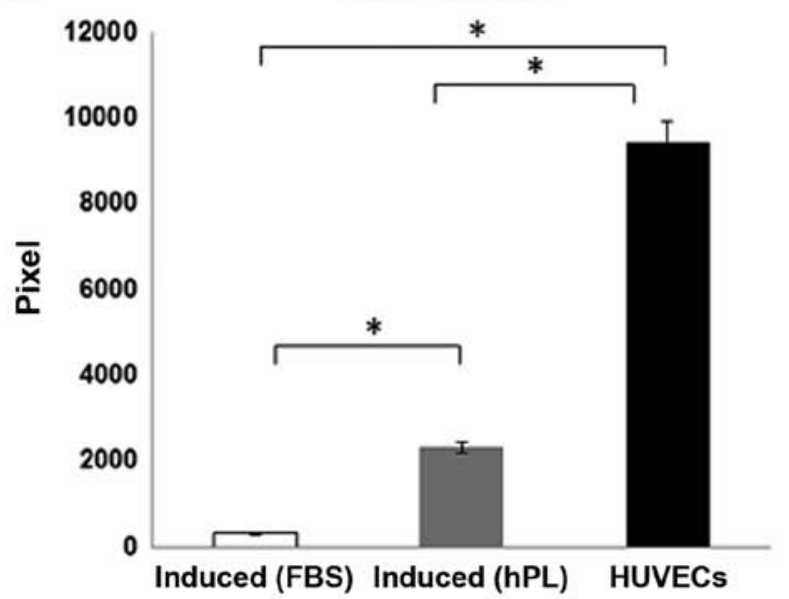

C
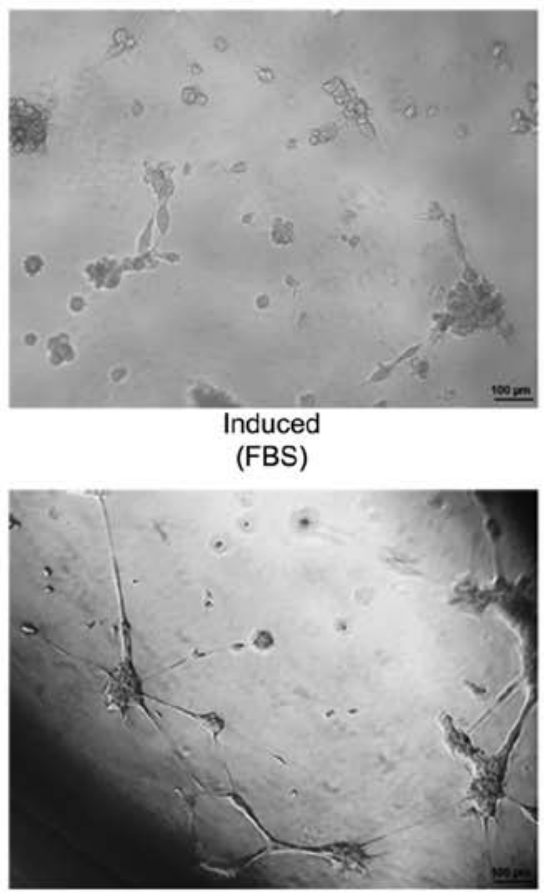

Induced

(hPL)

Mesh number

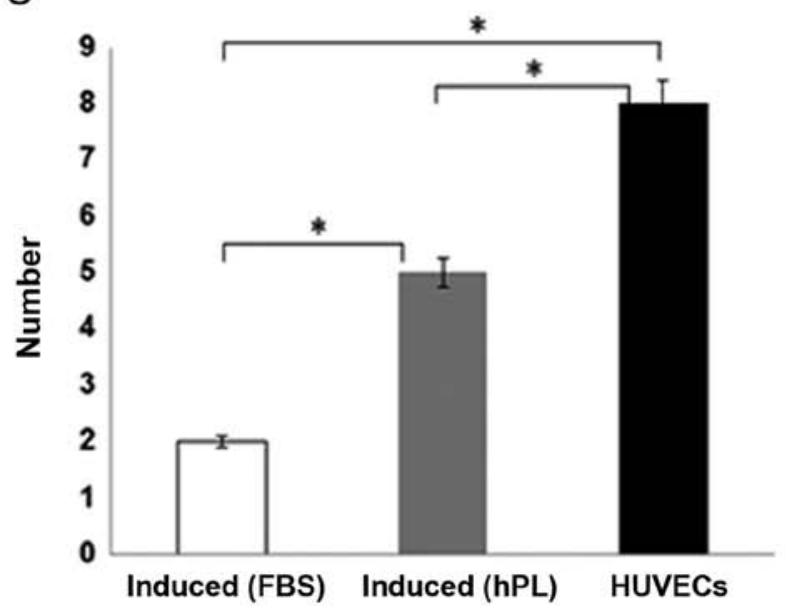

Figure 8. Ability of FBS-supplemented non-induced cells, FBS-supplemented induced cells, hPL-supplemented non-induced cell, hPL-supplemented induced cells and HUVECs to form a network-like structure. (A) Comparison of (B) total mesh area and (C) mesh number between FBS-supplemented induced cells, hPL-supplemented induced cells and HUVECs (magnification, x20; scale bar, $100 \mu \mathrm{m}$ ). The Kruskal-Wallis test and post hoc analysis were used to analyze the results of this experiment "P<0.05. hPL, human platelet lysate; HUVECs, human umbilical vein endothelial cell.

colony forming cells mediated by growth factors, including VEGF, bFGF and PDGF, leading to the formation of capillary network $(30,34)$. Angiogenic effects can be activated via the ERK1/2 pathway and the angiopoietin-1/Tie2 pathway in response to the level of VEGF (35). Moreover, the effect of hPL treatment on endothelial cells can stimulate the ERK1/2 
pathway to enhance wound healing, cell proliferation and vessel growth (36). The data in the present study indicate that hPL has a role in the differentiation of hAF-MSCs, as they exhibited some endothelial specific markers (vWF, VEGFR2 and eNOS) and formed capillary-like structures. Therefore, the use of pooled hPL may be applied in xenogenic-free approaches and strategies to improve vascularization.

A limitation of the present study is the requirement for more information about the concentration of growth factors in hPL. The identification and quantification of the various growth factors in hPL using ELISA is necessary. The effect of other growth factors that may synergize with VEGF should also be considered. In addition, a larger expression profile should be carried out. It is also important to identify the factors, and their concentrations, present in FBS and hPL. Finally, it is also important to elucidate the role of specific growth factors in the endothelial differentiation pathway.

In conclusion, the present study has demonstrated that hAF-MSCs can be cultured in hPL-supplemented media while maintaining the proliferation and immune phenotype, as well as increasing the endothelial differentiation potential. This suggests that hPL may be used as an alternative to FBS. hPL is also associated with a high degree of safety when used in clinical grade-cell expansion and the treatment of vascular disease.

\section{Acknowledgements}

All human amniotic fluid cells were provided by the Human Genetic Laboratory, Department of Anatomy and the Thailand Excellence Center for Tissue Engineering and Stem Cells, Department of Biochemistry, Faculty of Medicine, Chiang Mai University. The authors would like to thank the blood bank of Maharaj Nakorn Chiang Mai Hospital for supplying the platelet concentrates.

\section{Funding}

This study was funded by the Faculty of Medicine, Chiang Mai University, Chiang Mai, Thailand (grant no. ANA-2561-05343).

\section{Availability of data and materials}

All data generated or analyzed during this study are included in this published article.

\section{Authors' contributions}

WT and SA conceived the experiments, and PP designed the experiments. KB, CP and NP made substantial contributions to the design of the study. SN analyzed and interpreted the data. RM and TL collected and analyzed the data. CT performed the experiments. The paper was drafted by WT and the manuscript was approved by all authors.

\section{Ethics approval and consent to participate}

Written informed consent was obtained and the protocol employed was approved by the Ethics Committee of the Faculty of Medicine, Chiang Mai University on March 13th 2018 (no. ANA-2561-05343).

\section{Patient consent for publication}

Not applicable.

\section{Competing interests}

The authors declare that they have no competing interests.

\section{References}

1. Cananzi M, Atala A and De Coppi P: Stem cells derived from amniotic fluid: New potentials in regenerative medicine. Reprod Biomed Online 18 (Suppl 1): S17-S27, 2009.

2. Antonucci I, Stuppia L, Kaneko Y, Yu S, Tajiri N, Bae EC, Chheda SH, Weinbren NL and Borlongan CV: Amniotic fluid as a rich source of mesenchymal stromal cells for transplantation therapy. Cell Transplant 20: 789-795, 2011.

3. Zhou J, Wang D, Liang T, Guo Q and Zhang G: Amniotic fluid-derived mesenchymal stem cells: Characteristics and therapeutic applications. Arch Gynecol Obstet 290: 223-231, 2014.

4. Wei X, Yang X, Han ZP, Qu FE, Shao L and Shi YF: Mesenchymal stem cells: A new trend for cell therapy. Acta Pharmacol Sin 34: 747-754, 2013.

5. Liu JW, Dunoyer-Geindre S, Serre-Beinier V, Mai G, Lambert JF, Fish RJ, Pernod G, Buehler L, Bounameaux H and Kruithof EK: Characterization of endothelial-like cells derived from human mesenchymal stem cells. J Thromb Haemost 5: 826-834, 2007.

6. Lidong G, Shaoqing L, Yunfang W, Huimin Y, Daqing L, Lijuan H, Cixian B, Fang Y and Xue N: In vitro differentiation of human adipose-derived mesenchymal stem cells into endothelial-like cells. Chin Sci Bull 51: 1863-1868, 2006.

7. Witzeneder K, Lindenmair A, Gabriela C, Höllera K, Theißa D, Redlb $\mathrm{H}$ and Hennerbichler S: Human-derived alternatives to fetal bovine serum in cell culture. Transfus Med Hemother 40: 417-423, 2013.

8. Rauch C, Feifel E, Amann EM, Spötl HP, Schennach H, Pfaller W and Gstraunthaler G: Alternatives to the use of fetal bovine serum: Human platelet lysates as a serum substitute in cell culture media. ALTEX 28: 305-316, 2011.

9. Hemeda H, Giebel B and Wagner W: Evaluation of human platelet lysate versus fetal bovine serum for culture of mesenchymal stromal cells. Cytotherapy 16: 170-180, 2014.

10. Naaijkens B, Niessen HW, Prins HJ, Krijnen PA, Kokhuis TJ, de Jong N, Van Hinsbergh VW, Kamp O, Helder MN, Musters RJ, et al: Human platelet lysate as a fetal bovine serum substitute improves human adipose-derived stromal cell culture for future cardiac repair applications. Cell Tissue Res 348: 119-130, 2012.

11. Antoninus AA, Widowati W, Wijaya L, Agustina D, Puradisastra S, Sumitro SB, Widodo MA and Bachtiar I: Human platelet lysate enhances the proliferation of Wharton's jelly-derived mesenchymal stem cells. Biomarkers Genomic Med 7: 87-97, 2015.

12. Bieback K, Hecker A, Kocaömer A, Lannert H, Schallmoser K, Strunk D and Klüter H: Human alternatives to fetal bovine serum for the expansion of mesenchymal stromal cells from bone marrow. Stem Cells 27: 2331-2341, 2009.

13. Doucet C, Ernou I, Zhang Y, Llense JR, Begot L, Holy X and Lataillade JJ: Platelet lysates promote mesenchymal stem cell expansion: A safety substitute for animal serum in cell-based therapy applications. J Cell Physiol 205: 228-236, 2005.

14. Avanzini MA, Bernardo ME, Cometa AM,Perotti C,Zaffaroni N, Novara F, Visai L, Moretta A, Del Fante C, Villa R, et al: Generation of mesenchymal stromal cells in the presence of platelet lysate: A phenotypic and functional comparison of umbilical cord blood- and bone marrow-derived progenitors. Haematologica 94: 1649-1660, 2009.

15. Homayouni Moghadam F, Tayebi T, Moradi A, Nadri H, Barzegar K and Eslami G: Treatment with platelet lysate induces endothelial differentiation of bone marrow mesenchymal stem cells under fluid shear stress. EXCLI J 13: 638-649, 2014.

16. Soltis RD, Hasz D, Morris MJ and Wilson ID: The effect of heat inactivation of serum on aggregation of immunoglobulins. Immunology 36: 37-45, 1979. 
17. Tancharoen W, Aungsuchawan S, Pothacharoen P, Markmee R, Narakornsak S, Kieodee J, Boonma $\mathrm{N}$ and Tasuya W: Differentiation of mesenchymal stem cells from human amniotic fluid to vascular endothelial cells. Acta Histochem 119: 113-121, 2017.

18. Livak KJ and Schmittgen TD: Analysis of relative gene expression data using real-time quantitative PCR and the 2(-Delta Delta C(T)) method. Methods 25: 402-408, 2001.

19. Fei X, Jiang S, Zhang S, Li Y, Ge J, He B, Goldstein S and Ruiz G: Isolation, culture, and identification of amniotic fluid-derived mesenchymal stem cells. Cell Biochem Biophys 67: 689-694, 2013.

20. Naskou MC, Sumner SM, Chocallo A, Kemelmakher H, Thoresen M, Copland I, Galipeau J and Peroni JF: Platelet lysate as a novel serum-free media supplement for the culture of equine bone marrow-derived mesenchymal stem cells. Stem Cell Res Ther 9: 75, 2018.

21. Schallmoser K, Bartmann C, Rohde E, Reinisch A, Kashofe K, Stadelmeyer E, Drexler C, Lanzer G, Linkesch W and Strunk D: Human platelet lysate can replace fetal bovine serum for clinical-scale expansion of functional mesenchymal stromal cells. Transfusion 47: 1436-1446, 2007.

22. Fekete N, Gadelorge M, Fürst D, Maurer C, Dausend J, Fleury-Cappellesso S, Mailänder V, Lotfi R, Ignatius A, Sensebé L, et al: Platelet lysate from whole blood-derived pooled platelet concentrates and apheresis-derived platelet concentrates for the isolation and expansion of human bone marrow mesenchymal stromal cells: Production process, content and identifi cation of active components. Cytotherapy 14: 540-554, 2012.

23. Burnouf T, Strunk D, Koh MB and Schallmoser K: Human platelet lysate: Replacing fetal bovine serum as a gold standard for human cell propagation? Biomaterials 76: 371-387, 2016.

24. Lykov AP, Bondarenko NA, Surovtseva MA, Kim II, Poveshchenko OV, Pokushalov EA and Konenkov VI: Comparative effects of platelet-rich plasma, platelet lysate, and fetal calf serum on mesenchymal stem cells. Bull Exp Biol Med 163: 757-760, 2017.

25. Lohmann M, Walenda G, Hemeda H, Joussen S, Drescher W, Jockenhoevel S, Hutschenreuter G, Zenke M and Wagner W: Donor age of human platelet lysate affects proliferation and differentiation of mesenchymal stem cells. PLoS One 7: e37839, 2012.

26. Dominici M, Le Blanc K, Mueller I, Slaper-Cortenbach I, Marini F, Krause D, Deans R, Keating A, Prockop DJ and Horwitz E: Minimal criteria for defining multipotent mesenchymal stromal cells. The international society for cellular therapy position statement. Cytotherapy 8: 315-317, 2006.

27. Narakornsak S, Poovachiranon N, Peerapapong L, Pothacharoen P and Aungsuchawan S: Mesenchymal stem cells differentiated into chondrocyte-Like cells. Acta Histochem 118: 418-429, 2016
28. Laowanitwattana T, Aungsuchawan S, Narakornsak S, Markmee R, Tancharoen W, Keawdee J, Boonma N, Tasuya W, Peerapapong L, Pangjaidee N and Pothacharoen P: Osteoblastic differentiation potential of human amniotic fluid-derived mesenchymal stem cells in different culture conditions. Acta Histochem 120: 701-712, 2018.

29. Ben Azouna N, Jenhani F, Regaya Z, Berraeis L, Ben Othman T, Ducrocq E and Domenech J: Phenotypical and functional characteristics of mesenchymal stem cells from bone marrow: Comparison of culture using different media supplemented with human platelet lysate or fetal bovine serum. Stem Cell Res Ther 3: 6, 2012 .

30. Kim H, Prasain N, Vemula S, Ferkowicz MJ, Yoshimoto M, Voytik-Harbin SL and Yoder MC: Human platelet lysate improves human cord blood derived ECFC survival and vasculogenesis in three dimensional (3D) collagen matrices. Microvasc Res 101:72-81, 2015.

31. Jazayeri M, Allameh A, Soleimani M, Jazayeri SH, Kaviani S and Kazemnejad S: Capillary network formation by endothelial cells differentiated from human bone marrow mesenchymal stem cells. Iran J Biotechnol 6: 29-35, 2008.

32. Gang EJ, Jeong JA, Han S, Yan Q, Jeon CJ and Kim H: In vitro endothelial potential of human UC blood-derived mesenchymal stem cells. Cytotherapy 8: 215-227, 2006.

33. Quirici N, Soligo D, Caneva L, Servida F, Bossolasco P and Deliliers GL: Differentiation and expansion of endothelial cells from human bone marrow CD133(+) cells. Br J Haematol 115: 186-194, 2001

34. Fortunato TM, Beltrami C, Emanueli C, De Bank PA and Pula G: Platelet lysate gel and endothelial progenitors stimulate microvascular network formation in vitro: Tissue engineering implications. Sci Rep 6: 25326, 2016.

35. Hofbauer P, Riedl S, Witzeneder K, Hildner F, Wolbank S, Groeger M, Gabriel C, Redl H and Holnthoner W: Human platelet lysate is a feasible candidate to replace fetal calf serum as medium supplement for blood vascular and lymphatic endothelial cells. Cytotherapy 16: 1238-1244, 2014.

36. Barsotti MC, Losi P, Briganti E, Sanguinetti E, Magera A, Al Kayal TA, Feriani R, Di Stefano R and Soldani G: Effect of platelet lysate on human cells involved in different phases of wound healing. PLoS One 8: e84753, 2013.

This work is licensed under a Creative Commons Attribution-NonCommercial-NoDerivatives 4.0 International (CC BY-NC-ND 4.0) License. 